\title{
EARLY OPERATIONS FOR THE CLOSURE OF CLEFT PALATE.
}

\author{
By Truman W. Brophy, M. D., D. D. S. \\ OF CHICAGO.
}

Operations for the closure of cleft palate should be performed in early infancy. In making this statement, I fully realize that my views are not in accord with those of many distinguished surgeons, but clinical experience has demonstrated that what I presented to the profession a few years ago as a theory in regard to palate operations, has become a well-established practice. It is well known that many infants born with this defect die within a short time after birth, when the cleft is of a marked character. Lawson Tait says that one-half of the children born with extensive clefts, die from starvation within a few days after birth.

In the complication of hare-lip with cleft palate, the practice has almost invariably been to operate upon the lip, and allow the fissure of the palate to remain unclosed. This, in my opinion, is a mistake. The palate should be operated upon first, for various reasons.

1. The fissure of the lip enables the operator to gain a little more room in which to work.

2. The closure of the palate is an operation attended with great difficulties in its performance; besides, the closure of the cleft through the alveolar process, if it exists, may be more surely accomplished, when fully exposed to view through the divided lip than when covered, as it would be subsequent to the closure of the lip.

It is unnecessary to say that the bones are soft and will easily yield in early infancy. The tissues unite kindly, and the shock following the operation is not so great if performed within the first month, as it would be later in childhood. It is a well known fact that the nervous system of a child is not so well developed in early infancy as it is later; hence, one of the advantages of performing this operation before the nervous system has developed to a point which would subject the child to a more severe shock. The operation, therefore, should be performed as soon as the functions of the organs of the body are well 
established. This may be within the first week, or any time within the first month.

3. After the operation the child will be better nourished.

4. If performed in early infancy, the muscles of the palate will be brought into action and will be developed, whereas, if the muscles are not thus put into use, they will atrophy, and later it will be found, as is often the case, that there is insufficient tissue to restore the palate to its normal form. It is scarcely necessary to state that muscular tissue which is not subject to action and use will not develop normally.

5. One of the most important reasons why we should operate in early infancy is, that the parts operated upon early not only develop well, but the child when arriving at the age when articulation in the form of speech is attempted, finds that he can speak as other children do, and does not acquire the habit of articulating through the nasal passage, which is characteristic of this deformity, for the nasal accent, when once acquired, cannot be easily corrected. Fissures or cleft of the palate are almost invariably accompanied with pharyngitis.

6. The operation on the hard palate can be more easily and successfully accomplished before the calcification of the bone is far advanced, and also before the eruption of the teeth; if made subsequent to the eruption of the teeth, it is always attended with more difficulty, and the results are less satisfactory.

The operation is as follows: Introduce the oral speculum and vivify the edges of the fissure; do it thoroughly. A mere scraping of the mucous membrane will never suffice to bring about union which will be permanent and satisfactory. Trim well the opposing surfaces of the bone on the hard palate. If this be well done, it will secure a sufsufficient exudate to make the operation a successful one in this respect. The knife will easily cut through the soft bone of the hard palate and the alveolar process of young patients. Then raise the cheek, and well back toward the posterior extremity of the hard palate, just back of the molar process, and high enough to escape all danger of not being above the palatal plate of the bone, insert a large braided silk suture, carrying it through the substance of the bone, so that it will come out at a corresponding position upon the opposite side. It is often difficult to pass the suture through to the opposite side, and under such 
circumstances the needle, with eye in point, may be carried from the buccal surface of the bone to the median line, the stitch taken up with a tenaculum, and then the needle inserted from the opposite side, the thread then to be united and carried through. The silk suture is more easily introduced by the needle, but a wire suture of silver should be substituted for it, and drawn through in its place, and this wire may be doubled, in case the condition of the parts and the tension upon the tissues necessary to approximate them, seem to require it.

Nearer the front portion of the palate, insert another wire, carrying it through the substance of the bone above the palatal plates, and out through the other side, in a position corresponding to the place of entrance. Thus we shall have one wire passing over the palate in front of the malar process of the bone, and another behind it.

The next step is to take a lead button, moulded to fit the convexity 'of the part, and long enough to pass beyond the exit of the wire sutures, so that they will pass through it. Have it provided with eye-holes, through which are passed the protruded ends of the wires upon each side, and then twisted. These are heavy tension sutures, and once approximated, the parts cannot be separated by the patient. If we are unable to close the fissure with these wires, if from lack of tissue or from firm resistance of the parts it cannot be done, there is a further method to be employed which will obviate these difficulties. After the cheek is well raised, divide the mucous membrane just over the malar process. Here insert a knife in a horizontal direction, and when well inserted, sweep the handle around from one side to the other, as from behind forward. In this way a maximum amount of bone is divided and a minimum amount of the mucous membrane. This done on each side, the bone can very readily be moved toward the middle line. Having thus divided the bones upon either side, the wire sutures passing through the lead buttons may again be twisted, and the cleft of the hard palate will be easily closed by approximation of the two sides. The incision in the mucous membrane, in making. the separation of the bones, should be as small as possible, for the reason that this membrane must serve to retain the bones in proximity, or to hold them nearly together. If, after the parts are approximated, they are kept antiseptically clean, the bones will kindly unite and the palate will 
be restored, so that its full function will be performed. Separation of the bone is attended with little hemorrhage, and the parts do not usually cause more inconvenience to the patient than the ordinary operation of lifting the hard palate, according to the practice of Ferguson.

The germs of the teeth are sometimes disturbed, as I have found later, when they were erupted, that certain teeth were imperfectly formed. This applies to the molars of the temporary set, but the germs of the permanent teeth may also be disturbed, and the teeth made imperfect by this procedure, but the dental defects are of little importance when offset by the benefits of being relieved of so great a deformity. The palate, too, may be contracted to an abnormal extent; and yet it is a well known fact that the alveolar process develops with the eruption of the teeth, and my experience has convinced me that in mouths thus treated, the upper teeth, when they erupt, oppose in a normal way, those of the lower jaw. If, however, the superior arch should be abnormally contracted, the means well known to the modern dentist may be employed to widen it.

After the approximation of the edges in the manner I have described, the parts should be thoroughly dried, the edges of the wound carefully examined, and if need be, some fine sutures inserted here and there, to ensure perfect co-aptation of the parts. The after-treatment is very simple, consisting solely in antiseptic cleanliness, nourishing of the patient upon liquid food, the prevention of disturbance of the parts of the child, or of the introduction into the mouth of anything that might interfere with the sutures. Abrasions of the mucous membrane caused by the buttons, need not disturb the operator, for they are usually slight. 